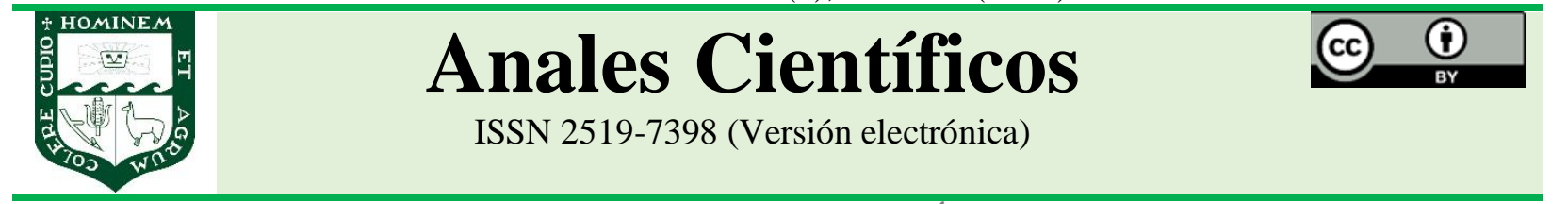

ARTÍCULO ORIGINAL - RESEARCH ARTICLE

http://dx.doi.org/10.21704/ac.v82i1.1748

\title{
DEFORESTACIÓN POR ACTIVIDAD MINERA EN EL DEPARTAMENTO DE MADRE DE DIOS-PERÚ PARA LOS AÑOS 2000 Y 2017
}

\section{Mining deforestation in Madre de Dios - Peru for years 2000 and 2017}

\author{
Lasmit Cerón C. ${ }^{1,2 *(1)}$; Natalia Miranda S. ${ }^{1,3}$;0; Ethel Rubin-de-Celis L. ${ }^{1,4}(\mathbb{C})$ \\ ${ }^{1}$ Sala de Observación Perú de la Organización del Tratado de Cooperación Amazónica, 15024, Lima. \\ ${ }^{2}$ Facultad de Ciencias Forestales, Universidad Nacional Agraria La Molina, La Molina, 15024, Lima, Perú. \\ *E-mail: 20091044@lamolina.edu.pe
}

Recivido: 09/03/202020; Aceptado: 14/04/2021; Publicado: 31/08/2021

\begin{abstract}
Since the 1980s gold mining in Madre de Dios, Perú has been the main deforestation driver generating severe socioeconomic and ecological impacts. The present research quantifies this activity in 2000 and 2017 by decision tree algorithms applied on a supervised classification of satellite images from LANDSAT 5 and LANDSAT 8 sensors. Results reveal an increase of 47657,43 ha of deforestation due to gold mining between 2000 and 2017. Categorized areas showed an increase of 42,52\% (35 183,45 ha), with the greatest impact on the native communities, concessions for reforestation and buffer zones of Protected Natural Areas. It is worth mentioning that there were 1 139,77 ha by 2000 that presented mining activity and were recovered by 2017 . Validation by experts confirmed up to $93 \%$ of the detections with Kappa index. Decision tree methodology is direct, with quick processes and accurate results.
\end{abstract}

Keywords: Gold mining; Landsat; Decision trees algorithms; Territorial categories; Peruvian Amazon

\section{RESUMEN}

Desde la década de los 80 la minería aurífera en Madre de Dios ha sido el principal impulsor de la deforestación generando impactos socioeconómicos y ecológicos graves. El presente estudio tiene como objetivo cuantificar la minería aurífera en el departamento de Madre de Dios, Perú en los años 2000 y 2017, empleando una clasificación supervisada mediante algoritmos de árboles de decisión en imágenes satelitales de los sensores LANDSAT 5 y LANDSAT 8. Los resultados muestran un incremento de 47657,43 ha con actividad minera entre el año 2000 y 2017. Asimismo, en las zonas categorizadas se cuantificó un aumento de $42,52 \%$ equivalente a 35183,45 ha, siendo las comunidades nativas, concesiones para reforestación y zonas de amortiguamiento de Áreas Naturales Protegidas las áreas más afectadas. Se identificaron 1 139,77 ha que en el año 2000 presentaron actividad minera y recuperaron su cobertura vegetal para el año 2017. El resultado fue validado mediante juicio de expertos y se obtuvo un índice de Kappa de 93\% de acierto. La metodología de árboles de decisión es directa, con procesos rápidos y resultados precisos.

Palabras clave: Minería aurífera; Landsat; Árboles de decisión; Categorías territoriales; Amazonía peruana 


\section{Forma de citar el artículo (Formato APA):}

Cerón, L.; Miranda, N. \& Rubin-de-Celis, E. (2021). Deforestación por actividad minera en el departamento de Madre de Dios-Perú para los años 2000 y 2017. Anales Científicos. 82(1), 122-129. http://dx.doi.org/10.21704/ac.v82i1.1748

Autor de correspondencia (*): Lasmit Cerón C. Email: 20091044@lamolina.edu.pe

(C) Los autores. Publicado por la Universidad Nacional Agraria La Molina.

This is an open access article under the CC BY

\section{INTRODUCCIÓN}

La región amazónica de Madre de Dios es considerada como una de las 25 áreas de mayor diversidad del planeta: la Reserva Nacional de Tambopata y el Parque Nacional del Manu, junto al Bahuaja Sonene, forman parte del Corredor de Conservación Vilcabamba Amboro (De Echave, 2016). A su vez, juega un rol fundamental en la conservación de la riqueza paisajística y recursos renovables del mundo (Rodrigues et al., 2004). Sin embargo, a lo largo del tiempo los ecosistemas de los bosques amazónicos han sido alterados por actividades extractivas, para obtener productos de origen maderable, no maderable, agricultura, minería, entre otros (Brack, 2000). La demanda creciente de estos productos está ocasionando una deforestación alarmante que requiere información actualizada respecto a la condición y posibles cambios en el uso del suelo y vegetación en la Amazonía (Dourojeanni, 2006).

Un problema a nivel nacional, que compromete uno de los bosques más representativos de la Amazonía, es la deforestación causada por la minería aurífera. En el Perú, la región donde se produce la mayor cantidad de extracción de oro es el departamento de Madre de Dios, donde la minería constituye la principal actividad económica. El mercurio utilizado en esta actividad es depositado en los lechos de los ríos causando la contaminación de la cadena trófica de la fauna, esta fauna incluye a los peces que son parte de la dieta diaria de los habitantes. Resultando en consecuencias graves en la salud de los pobladores y, colateralmente, en la deforestación de los bosques, conflictos de derechos de uso de la tierra, asuntos sociales y culturales (Arana \& Chang, 2005; Medina et al., 2007; Quintanilla \& Maguiña, 2008; Brack et al., 2011).

Ante la necesidad de realizar un monitoreo de los bosques de la Amazonía, la Organización del Tratado de Cooperación Amazónica (OTCA) implementó en octubre del 2013 la segunda fase del Proyecto: "Monitoreo de la Cobertura Forestal en la Región Amazónica", creando con sus puntos focales peruanos Servicio Nacional Forestal y de Fauna silvestre (SERFOR) - Ministerio de Agricultura (MINAGRI) y Programa Nacional de Conservación de Bosques y Cambio Climático (PNCBCC) Ministerio del Ambiente (MINAM), la Sala de Observación de Perú (OTCA, 2016). Para este tipo de proyectos se emplean sistemas de información geográfica y teledetección como herramientas que permiten estudiar zonas extensas, remotas y difíciles de acceder en campo, como lo señalan Moran \& Brondizio (1994). En estudios sobre actividad minera en la Amazonía peruana, diversos autores han aplicado dichas herramientas con resultados precisos (Swenson et al., 2011; Asner et al., 2013; Caballero et al, 2018).

Swenson et al. (2011) utilizaron datos satelitales gratuitos de Landsat 5 y técnicas de interpretación de imágenes usadas en teledetección para mapear áreas con minería aurífera en las tres principales zonas de actividad en Madre de Dios: Guacamayo o La Pampa, Colorado-Puquiri o Delta y Huepetuhe durante los años 2003, 2006 y 2009. Los resultados, no fueron validados, pero indicaron que las tres áreas constituyeron 15500 ha en el año 2009 y que las dos últimas se ubican parcialmente en las zonas de amortiguamiento de la Reserva Comunal Amarakaeri y el Parque Nacional del Manu.

El estudio de Asner et al. (2013) sobre la minería aurífera en Madre de Dios emplea herramientas SIG con imágenes Landsat 5 y Landsat 7 para el periodo 1999 - 2012. Según los resultados que obtuvieron, para el año 1999 existían 10000 ha de actividad minera, aumentando a 32371 ha en el año 2009 y a 50000 ha en el año 2012. Adicionalmente, estimaron ratios promedio de incremento anual de la actividad minera de 2166 ha/año para los años previos al 2008 y de 6145 ha/año para el periodo 2008 - 2012. Los autores emplean una metodología 
de validación que confirma el $94 \%$ de las detecciones de su clasificación y además considera áreas con operaciones mineras a pequeña escala, las cuales no fueron consideradas en el estudio de Swenson et al. (2011), donde limitaron el análisis a las tres principales áreas antes mencionadas.

El reporte de Finer et al. (2016) sobre actividad minera en Madre de Dios para el periodo 2012 2016 indica, en base a resultados de tres estudios previos, que en el año 2016 el área con actividad minera fue de 62500 ha. Además, se hace mención y se ilustra la intrusión de la minería en las áreas de amortiguamiento de la Reserva Nacional de Tambopata, el Parque Nacional Bahuaja Sonene y la Reserva Comunal Amarakaeri.

Estudios más recientes como los de Asner y Tupayachi (2017) y Caballero et al. (2018), muestran resultados de un análisis multitemporal con imágenes Landsat 5, Landsat 7 y Landsat 8, el primero para el periodo 1999 - 2016 y el segundo amplía el periodo desde 1985 hasta el 2017. Ambos autores emplean la misma metodología y validación, sin embargo, Caballero et al. (2018) implementa algunos ajustes técnicos en el procesamiento de las imágenes que afinan la exactitud de sus resultados.

Asner y Tupayachi (2017) estimaron que para el año 1999 la actividad minera comprendía 9408 ha concentradas en su mayoría en la zona de Huepetuhe, y para el año 2016 el área aumentó a 68 228 ha. El estudio además hace especial énfasis en la Reserva Nacional de Tambopata, indicando que sólo en su zona de amortiguamiento, la deforestación por minería alcanzó 30047 ha para el año 2016, lo que representa el 44\% del total de la actividad minera en Madre de Dios.

Caballero et al. (2018) muestran como resultado un total de 95751 ha con actividad minera que fueron temporalmente distribuidas en dos periodos, 31165 ha entre los años 1985 - 2009 y, 64586 ha durante los años 2010 - 2017. También indican que las áreas La Pampa, Delta y Huepetuhe al 2017 abarcan 47 113 ha con actividad minera y las áreas con operaciones mineras de pequeña escala constituyeron el $65 \%$ del total con 48636 ha, superando a las grandes minas. Cabe mencionar que el análisis determina a la actividad minera como principal causa de deforestación (60\%) en Madre de Dios desde el 2010 hasta el 2015. Sin embargo, desde el 2016, otros factores impulsores incrementaron sustancialmente, reduciendo la contribución relativa de la minería a un $38 \%$.

El objetivo de la investigación fue identificar los cambios en la cobertura forestal causados por minería aurífera en el departamento de Madre de Dios durante los años 2000 y 2017, empleando una metodología simple de clasificación supervisada basada en árboles de decisión, con el fin de cuantificar el área afectada y analizar su distribución en las distintas categorías territoriales.

\section{MATERIALES Y MÉTODOS}

El departamento de Madre de Dios se ubica en la región sur oriental del territorio peruano, entre las coordenadas geográficas $9^{\circ} 57^{\prime}$ y $13^{\circ} 20^{\prime}$, latitud sur, $68^{\circ} 39^{\prime}$ y $72^{\circ} 31^{\prime}$, longitud oeste. Cuenta con una población de 141070 habitantes (INEI, 2017) y una superficie de 8530054 ha, la cual representa el 6,6\% del territorio peruano y comprende tres provincias: Tahuamanu, Manu y Tambopata (Osores et al., 2012).

La determinación de la deforestación causada por minería se determinó mediante la realización de mosaicos a partir de imágenes satelitales Landsat 5 sensor TM (año 2000) y Landsat 8 sensor OLI (año 2017), con resolución espacial de $30 \mathrm{~m}$ y obtenidas gratuitamente de la plataforma U.S. Geological Survey (USGS, 2018). Las escenas utilizadas se muestran en la Figura 1. Para el análisis se empleó cartografía base en formato vectorial del área de estudio: categorías territoriales oficiales del SERFOR (2017), y el límite político oficial del departamento de Madre de Dios del 2016 del Instituto Nacional de Estadística e Informática (INEI, 2016). 


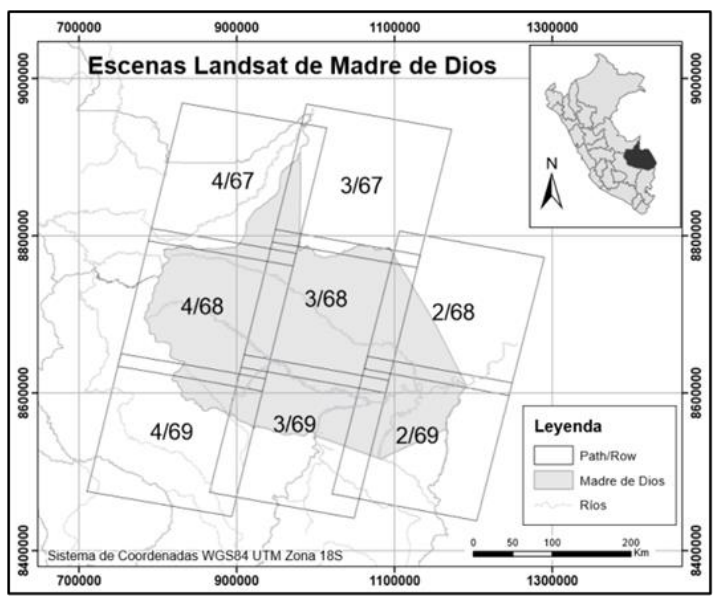

Figura 1. Ubicación del área de estudio.

El mosaico del año 2000 (Landsat 5 TM) fue elaborado con las bandas $5(1,55 \mu \mathrm{m}-1,75 \mu \mathrm{m}), 4$ $(0,76 \mu \mathrm{m}-0,9 \mu \mathrm{m})$ у $3(0,63 \mu \mathrm{m}-0,69 \mu \mathrm{m})$; mientras que el mosaico 2017 (Landsat 8 OLI), con las bandas $6(1,566 \mu \mathrm{m}-1,651 \mu \mathrm{m}), 5(0,851 \mu \mathrm{m}-$ $0,879 \mu \mathrm{m})$ у $4(0,636 \mu \mathrm{m}-0,673 \mu \mathrm{m})$ (USGS, 2018). El proceso de unión de bandas espectrales se realizó con el software ENVI Classic versión 5.3. No se aplicó ningún tipo de corrección a las imágenes previo a la elaboración del mosaico.

Para llevar a cabo la clasificación, primero se generaron manualmente áreas de entrenamiento en formato vectorial en función al tipo de cobertura observada. Las dos clases definidas y sus descripciones se señalan a continuación: Minería (M): actividad minera, pozas; y, No minería (NM): cultivos agrícolas, pastos, bosques, ríos, arenales, carreteras, zonas urbanas, nubes, sombras de nubes y áreas bandeadas.

Las áreas de entrenamiento con los atributos asignados fueron el insumo para la clasificación supervisada, método que identifica en la imagen satelital todas las áreas espectrales similares a las áreas de entrenamiento (Gutiérrez \& Jegat, 2005; Castillejo et al., 2009). La clasificación supervisada empleó árboles de decisión con el programa ERDAS Imagine versión 9.2 y See5 C-5, metodología utilizada en el Laboratorio de Teledetección Aplicada y SIG de la Facultad de Ciencias Forestales de la Universidad Nacional Agraria La Molina (UNALM). Este representa la relación que existe entre la decisión y sus atributos o variables (Vuletič et al., 1999; Díaz et al., 2005).

El resultado de la clasificación supervisada se obtuvo en formato raster y fue convertido a vector mediante el software ArcGis versión 10.5. Se realizó la edición manual de las clases por defecto o exceso de la clasificación para el año 2000 y 2017, luego se rasterizó para ajustar la forma de las clases al mosaico. Finalmente, se analizaron los resultados en base a las categorías territoriales del área de estudio.

La corroboración de los resultados fue mediante el empleo "la validez de contenido", evaluado a través de un juicio de expertos, el cual es definido como una opinión informada de personas con trayectoria en el tema que son reconocidas por otros, como expertos calificados, y que pueden dar información, evidencia, juicios y valoraciones (Escobar-Pérez \& Cuervo-Martínez, 2008).

En este proceso se evaluó un total de 40 puntos distribuidos por muestreo estratificado en el mosaico del año 2017, donde los estratos fueron las dos clases mencionadas. El evaluador se basó en su experiencia en campo y mediante la interpretación visual de imágenes de alta resolución obtenidas del programa Google Earth Pro identificó los puntos con actividad minera. Se elaboró una tabla de contingencia para calcular el coeficiente de kappa, con el fin de hallar el grado de concordancia inter-observador. Este coeficiente toma valores entre $-1 \mathrm{y}+1$, obteniéndose un mayor grado de concordancia inter-observador con valores cercanos a $+1 \mathrm{y}$, mayor grado de discordancia inter-observador con valores cercanos a -1 (Cerda \& Villarroel, 2008).

\section{RESULTADOS Y DISCUSIÓN}

Se generaron las capas de pérdida de cobertura vegetal por actividad minera para los años $2000 \mathrm{y}$ 2017, en la Figura 2 se muestran los mosaicos en color verdadero proyectados en coordenadas UTM Zona 18S. 


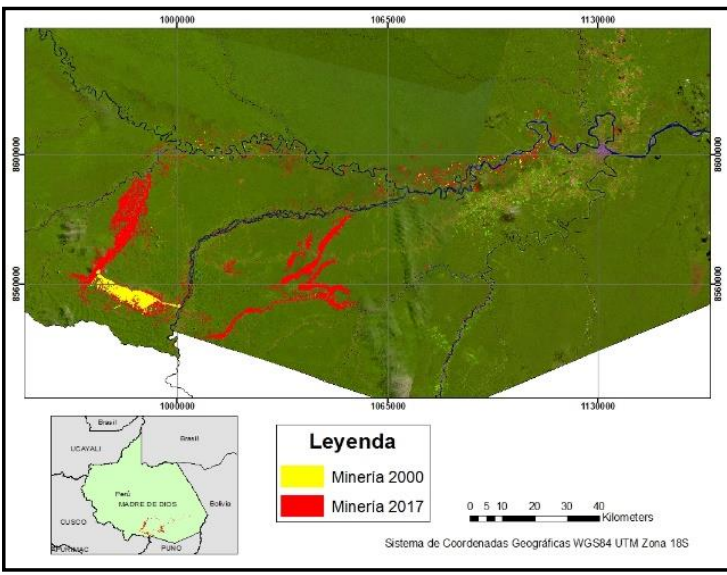

Figura 2. Capa de pérdida de cobertura por actividad minera - Años 2000 y 2017.

En el año 2000 la actividad minera fue de 9 041,04 ha ubicadas en la zona sureste de Madre de Dios, representando el $0,1 \%$ de la superficie del departamento. La minería en el año 2017 alcanzó 56 698,47 ha que representa el 0,66\% de Madre de Dios.

Según Osinfor (2016), para el año 2014 se estimó una pérdida de cobertura forestal de 47 189,33 ha equivalente al $0,55 \%$ del área de todo el departamento. Asimismo, según datos oficiales del Ministerio del Ambiente, de 6254 ha deforestadas en el año 2000 por actividad minera, en el año 2011 la cifra alcanzó 32750 ha y hasta el 2016 se cuantificaron más de 50000 ha deforestadas por minería de oro artesanal y en pequeña escala (AGSM), Echave (2016). En el estudio presentado por Finer et al. (2016), la cifra alcanzada para el año 2016 fue de 62500 ha.

El departamento de Madre de Dios presenta zonas categorizadas y no categorizadas (SERFOR, 2017). Dentro de la zona categorizada, los resultados muestran que en el año 2000 la categoría territorial más afectada por actividad minera fue Concesión no Maderable, con 1651,56 ha, seguidas de las categorías Concesión Maderable y Comunidades Nativas (Tabla 1).

Para el año 2014, las Concesiones Forestales que agrupan Concesión para Reforestación, Concesión no Maderable, Concesión Maderable, Concesión para Ecoturismo y Concesión para Conservación sumaron 11 532,41 ha, seguida de las Comunidades Nativas que representaron 8236,11 ha deforestadas (Osinfor, 2016).

En el año 2017, la categoría agrupada como Concesiones Forestales abarcó la mayor área con actividad minera alcanzando 18881,65 ha, seguida de la categoría Comunidades Nativas con 10 505,27 ha (Tabla 1). Asimismo, se puede observar que la actividad minera en la Zona no categorizada presenta un incremento de 12473,98 ha durante el periodo 2000-2017.

Tabla 1. Distribución de áreas con actividad minera por categorías territoriales.

\begin{tabular}{|c|c|c|c|c|c|}
\hline \multicolumn{2}{|r|}{ Categoría Territorial } & Año 2000 (ha) & $\%$ & Año 2017 (ha) & $\%$ \\
\hline & Zona categorizada & 2597,95 & 28,74 & 37781,4 & 66,64 \\
\hline \multirow{5}{*}{$\begin{array}{c}\text { Concesiones } \\
\text { Forestales }\end{array}$} & Concesión para Reforestación & 58,26 & 24,48 & 7943,51 & 33,30 \\
\hline & Concesión no Maderable & 1651,56 & & 3153,06 & \\
\hline & Concesión Maderable & 431,74 & & 4849,14 & \\
\hline & Concesión para Ecoturismo & 19,34 & & 2460,02 & \\
\hline & Concesión para Conservación & 52,78 & & 475,92 & \\
\hline \multicolumn{2}{|c|}{ Comunidades Nativas } & 343,56 & 3,80 & 10505,27 & 18,53 \\
\hline \multicolumn{2}{|l|}{ ANP nacional } & 4,06 & 0,04 & 1097,16 & 1,94 \\
\hline \multicolumn{2}{|c|}{ Zona de Amortiguamiento } & 36,66 & 0,41 & 7297,31 & 12,87 \\
\hline \multicolumn{2}{|r|}{ Zona no categorizada } & 6443,09 & 71,26 & 18917,07 & 33,36 \\
\hline \multicolumn{2}{|r|}{ Total } & 9041,04 & 100,00 & 56698,47 & 100,00 \\
\hline
\end{tabular}


En la Figura 3 se presenta la actividad minera por categoría territorial, donde la categoría Comunidades Nativas presenta un aumento de 10 161,71 ha, seguida de la categoría Concesión para Reforestación con 7885,25 ha, Zona de Amortiguamiento con 7 260,65 ha, Concesión Maderable con 4 417,4 ha y Concesión para Ecoturismo con 2 440,68 ha, en el mismo periodo. Las categorías que registraron un menor incremento de superficie con actividad minera fueron Concesión para Conservación con 423,14 ha, ANP nacional con 1 093,1 ha y Concesión no Maderable con 1501,5 ha durante el periodo evaluado.

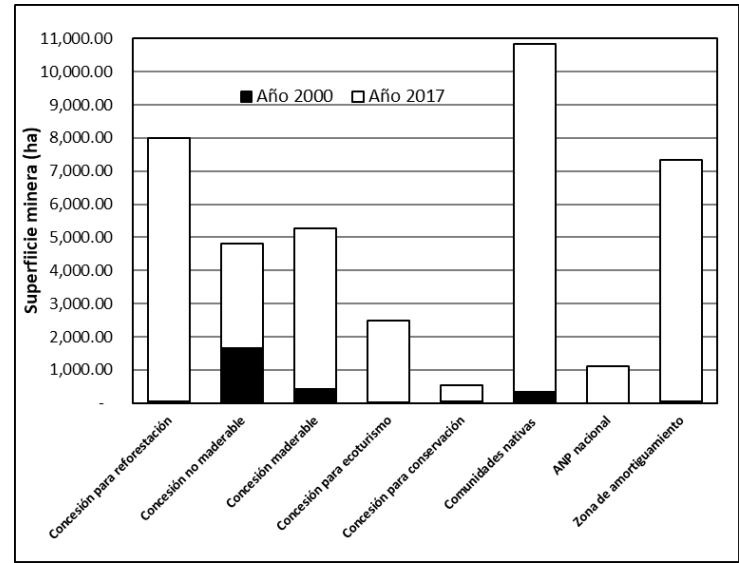

Figura 3. Actividad minera por categoría territorial en los años 2000 y 2017.

Adicionalmente se observó que 1 139,77 ha que fueron clasificadas como superficie con actividad minera en el año 2000 pasaron a ser zonas no mineras en el año 2017 (Tabla 2). Estas áreas que dejan de tener actividad minera pasan por diferentes sucesiones y tienen potencial para su posterior recuperación y restauración (Caballero et al., 2018).

Tabla 2. Cambio de cobertura durante el período $2000-2017$.

\begin{tabular}{|c|c|c|c|}
\hline & Año 2000 & $\begin{array}{l}\text { Año } \\
2017\end{array}$ & $\begin{array}{c}\text { Superficie } \\
\text { (ha) }\end{array}$ \\
\hline \multirow{3}{*}{$\begin{array}{l}\text { Cambio } \\
\text { de } \\
\text { cobertura }\end{array}$} & Minería & $\begin{array}{c}\text { No } \\
\text { minería }\end{array}$ & 1139,77 \\
\hline & Minería & Minería & 7901,27 \\
\hline & No minería & Minería & 47657,43 \\
\hline
\end{tabular}

La metodología empleada en este estudio muestra un trabajo sencillo y de bajo costo, pudiéndose monitorear la deforestación por minería, analizando de manera visual la huella espectral característica de la combinación de suelo desnudo y agua estancada. Los resultados obtenidos son similares a los de diversos autores (Swenson et al., 2011; Asner et al., 2013; Asner y Tupayachi, 2017; Caballero et al., 2018), que emplean técnicas más avanzadas de mapeo y validación. Asimismo, la fase final donde se editaron los resultados manualmente es una alternativa para editar áreas que pueden confundirse con las riberas y playas de los ríos.

El resultado de la verificación de la clasificación del año 2017 arrojó un acierto de 93\% mediante el índice de Kappa, el cual refleja la concordancia entre dos observadores. El valor obtenido de 0,93 , cercano a 1,00 , refleja el alto grado de concordancia entre los resultados de la clasificación realizada y el experto en teledetección y SIG (Cerda \& Villarroel, 2008), o un grado de acuerdo casi perfecto como lo clasifica Landis \& Koch (1977).

\section{CONCLUSIONES}

Es notable el incremento de las áreas afectadas por actividad minera entre el año 2000 y 2017, cifra que asciende a 47657 hectáreas, de las cuales el 74\% pertenecen a zonas categorizadas, cuya designación surgió con el fin de evitar la deforestación. Con esto se invita a analizar factores sociales, económicos y políticos que atiendan a los problemas derivados de la minería. Se recomienda la aplicación de la metodología en estudios futuros ya que permite detectar y cuantificar de forma directa cambios de cobertura y uso, asimismo se resalta la importancia del uso de imágenes de alta resolución para la etapa de validación.

\section{AGRADECIMIENTOS}

Al Ing. Carlos Salinas Montes Coordinador Regional Proyecto Monitoreo de la Deforestación, Aprovechamiento Forestal y Cambios en el Uso del Suelo en el Bosque Panamazónico de la OTCA quien hizo posible la realización de la investigación. A la Sala de Observación Perú que brindó las 
facilidades para realizar el estudio. A la Ing. Forestal Alejandra Zamora Rios, especialista en Teledetección y SIG de la Sala de Observación Perú - OTCA, quien realizó la validación mediante el Juicio de Expertos. A Erick Alor Milla y Lucia Bryson Cabrera por colaborar en la búsqueda de bibliografía para generar el artículo.

\section{Conflictos de intereses}

Los autores firmantes del presente trabajo de investigación declaran no tener ningún potencial conflicto de interés personal o económico con otras personas $\mathrm{u}$ organizaciones que puedan influir indebidamente con el presente manuscrito.

\section{Contribuciones de los autores}

Preparación y ejecución: LCC, NMS, ERC; Desarrollo de la metodología: LCC, NMS, ERC; Concepción y diseño: LCC, NMS, ERC; Edición del artículo: LCC, NMS, ERC; Supervisión del estudio: ERC.

\section{LITERATURA CITADA}

- Arana, M., \& Chang, J. (2005). Evaluación de los pasivos ambientales de la minería del río Malinowski en la reserva nacional Tambopata y su zona de amortiguamiento. Puerto Maldonado.

- Asner, G.P., Llactayo, W., Tupayachi, R., \& Ráez, E. (2013). Elevated rates of gold mining in the Amazon revealed through high-resolution monitoring. Proc. Natl. Acad. Sci. 2(7), 1-6.

- Asner, G.P., \& Tupayachi, R. (2017). Accelerated losses of protected forests from gold mining in the Peruvian Amazon. Environmental Research Letters, 12(9). https://doi.org/10.1088/1748-9326/aa7dab

- Brack, A. (2000). Biodiversidad amazónica y manejo de fauna silvestre. En: Manejo de fauna silvestre de la Amazonía. University of Florida UNDP/GEF, Instituto de Ecología. La Paz, Bolivia.

- Brack, A.; Ipenza, C. \& Álvarez, J. (2011). Informe Sobre Minería Aurífera En Madre De Dios Y Contaminación Con Mercurio -una Bomba De Tiempo. Ministerio del Ambiente, Departamento de Investigación, Lima.
- Caballero, J., Messinger, M., RománDañobeytia, F., Ascorra, C., Fernandez, L., \& Silman, M. (2018). Deforestation and Forest Degradation Due to Gold Mining in the Peruvian Amazon: A 34-Year Perspective. Remote Sensing, 10(12), 1903. https://doi.org/10.3390/rs10121903

- Castillejo, I.L., López, F., García, A., Peña, J.M., Jurado, M., Sánchez, M., \& González, M. (2009). Object and pixel-based analysis for mapping crops and their agro-environmental associated measures using QuickBird imagery. Computers and Electronics in Agriculture. 68(2), 207-215. https://doi.org/10.1016/j.compag.2009.06.004

- Cerda, J., \&Villarroel, P.L. (2008). Evaluación de la concordancia inter-observador en investigación pediátrica: Coeficiente de Kappa. Pontificia Universidad Católica de Chile.

- De Echave, J. (2016). La minería ilegal en Perú. Nueva Sociedad, 263(2), 131-144. Recuperado de https://www.minjus.gob.pe/wpcontent/uploads/2018/03/07_Mineria-ilegalArticulo-Peru-06.pdf

- Díaz, Z., Fernández, J., \& Segovia, M. (2005). El Algoritmo See5 versus la metodología Rough Set. Una aplicación a la predicción de la insolvencia en empresas españolas de seguros no-vida. Cuadernos de Estudios Empresariales (15), 179-198. Norteamérica. URL: https://revistas.ucm.es/index.php/CESE/article/ view/CESE0505110179A

- Dourojeanni, M. (2006). Estudio de caso sobre la carretera interoceánica en la Amazonía sur del Perú.

http://siar.minam.gob.pe/puno/sites/default/files /archivos/public/docs/437.pdf

- Escobar-Pérez, J., \& Cuervo-Martínez, Á. (2008). Validación de contenido y juicio de expertos: Una aproximación a su utilización. Avances en Medición, 6, 27-36. Colombia.

- Finer, M., Olexy, T., \& Novoa, S. (2016). Gold Mining Deforests 32000 Acres in southern Peruvian Amazon from 2013 to 2016. MAAP, 50. https://maaproject.org/2016/peru_gold

- Gutiérrez, J., \& Jegat, H. (2005). Uso de la teoría de lógica difusa en la clasificación de imágenes satelitales con coberturas mixtas: el caso urbano 
de Mérida, Venezuela. Interciencia, 30(5), 261266.

- INEI. [Instituto Nacional de Estadística e Informática]. (2016). Capa temática de los límites políticos del Perú. Consultado el 09 de octubre del 2017. Disponible en: https://www1.inei.gob.pe/contactenos/

- INEI. [Instituto Nacional de Estadística e Informática]. (2017). Censos Nacionales 2017: XII de Población, VII de Vivienda y III de Comunidades Indígenas. Consultado el 14 de abril del 2021.

- Landis, J., \& Koch, G. (1977). The measurement of observer agreement for categorical data. University of Michigan. Biometrics, 33(1), 159174. https://doi.org/10.2307/2529310.

- Medina, G., Arévalo, A.J., \& Quea, J.F. (2007). Estudio de Investigación de la Minería Ilegal en el Perú, Repercusiones para el sector minero y el país. Encuentro Empresarial: XXVIII Convención Minera.

- Moran, E.F., \& Brondizio, E. (1994). Integrating of Amazonian vegetation, land use, and satellite data. Bioscience, 44, 329-338.

- OSINFOR. (2016). Contribución en el monitoreo de la actividad minera en títulos habilitantes forestales, año 2014 - Departamento de Madre de Dios. Recuperado de https://www.osinfor.gob.pe/wpcontent/uploads/2016/06/Mineria-Madre-deDios-21-de-junio.pdf

- Osores, F., Rojas, J.E., \& Manrique, C.H. (2012). Minería informal e ilegal y contaminación con mercurio en Madre de Dios: Un problema de salud pública. Acta Médica Peruana. 29(1), 38-42. Accesado http://www.scielo.org.pe/scielo.php?script=sci_ arttext\&pid=S1728$59172012000100012 \& \operatorname{lng}=$ es\&tlng=es.

- OTCA. [Organización del Tratado de Cooperación Amazónica]. (2016). Monitoreo de la Cobertura Forestal en la Región Amazónica. Fundo Amazonia, 3(3), 1-8. Brasil. URL: http://www.fundoamazonia.gov.br/export/sites/ default/pt/.galleries/documentos/acervoprojetos-cartilhas-outros/OTCA-Boletin-3Proyecto-Monitoreo.pdf

- Quintanilla, T., \& Maguiña, K. (2008). Evaluación preliminar del impacto social de la minería aurífera aluvial en las zonas de Huepetuhe, Pukiri y Cabecera de Malinowsky, de la región Madre de Dios. Pro-Naturaleza y Movimiento El Pozo.

- Rodrigues, A.S., Andelman, S.J., Bakarr, M.I., Boitani, L., Brooks, T.M., Cowling, R.M., \& Yan, X. (2004). Effectiveness of the global protected area network in representing species diversity. Nature 428,640-643 https://doi.org/10.1038/nature02422.

- SERFOR. [Servicio Nacional Forestal y de Fauna Silvestre]. (2017). Capa temática de las categorías territoriales del Perú. Consultado el 09 de octubre del 2017. Disponible en: http://geo.serfor.gob.pe/geoserfor/

- Swenson, J.J., Carter, C.E., Domec, J.C., \& Delgado, C.I. (2011). Gold mining in the Peruvian Amazon: global prices, deforestation, and mercury imports. PLoS One. 6(4): e18875.

- USGS. [United States Geological Survey]. (2018). Band designations for the Landsat satellites. Disponible en https://www.usgs.gov/faqs/what-are-banddesignations-landsat-satellites?qtnews_science_products $=0$ \#qtnews_science_products

- Vuletič, S., Kern, J., Sonicki, Z., \& Ivankovič, D. (1999. Possibilities of see5 software in forecasting of life expectancy not achieving. In Serie: "Studies in Health Technology and Informatics". 68: 696-699. https://doi.org/10.3233/978-1-60750-912-7-696. 